\section{Dental Restoration: What Type of Filling?}

\section{'Effective Health Care' Bulletins Vol.5(2)}

NHS Centre For Reviews And Dissemination

http://www.york.ac.uk/inst/crd/ehc52.htm

The NHS Centre For Reviews And Dissemination at York, England has published a bulletin containing information on the effectiveness, cost and acceptability of health service interventions. The 12 page Bulletin which includes a comprehensive list of references has been produced by a research team at the University of York, and is funded by the Department of Health in England. It is based on a systematic review of the longevity of dental restorations commissioned by the Scottish Office and carried out by a team at the Dental School, University of Wales College of Medicine led by Professor Paul Dummer. The bulletin is written for dentists and other health care professionals and managers.

It starts with a summary of the significance of dental decay in terms of resources required to treat it. It reminds readers that tooth decay is one of the most common diseases and accounts for almost half of all tooth extractions. The treatment of tooth decay by the placement of simple, direct restorations (fillings) alone costs the NHS in England and Wales E173 million per year.

It also highlights the fact that dental restorations have a finite life. This is shown by the fact that over $60 \%$ of all restorative dentistry is for the replacement of restorations. It points out that new restorative materials are often marketed and introduced into practice with limited evidence on their long-term clinical performance.

Overall, amalgam is the direct restorative material of choice unless aesthetics are important. It lasts longest and is the cheapest. They note the concerns over safety of amalgam but reference the BDA's conclusion that extensive research has failed to establish any links between amalgam use and general ill health. The bulletin also points out that studies not included in the systematic review which used subjective criteria, and are more representative of the situation prevailing in general dental practice, make it clear that the longevity of amalgams and composite is considerably less than that achieved in the prospective studies included in the systematic review. Glass ionomer restorations have been in use for a much shorter time but they too have a high replacement rate in cross-sectional studies.

The newer generation dentine bonding agents for composite restorations use some form of acidic primer and have better retention rates than earlier generations. The use of cermet cements, and the composite and glass ionomer sandwich technique in class II cavities, had high failure rates and therefore cannot be recommended.

There is significant variation in decision making between dentists. Objective outcome measures might enable restorations to last longer before the decision to replace a filling was made. It is claimed that the likelihood of having a restoration replaced is more than doubled when a patient changes practitioners. Appropriate criteria for replacement of restorations are needed and dental schools should train dentists in their use in order to reduce unnecessary procelures and improve quality.

The longevity of restorations carried out in the better quality research studies suggests that routine clinical practice may be producing sub-optimal results. Work is needed to establish means of improving the quality of routine practice, putting in place incentives to promote cost-effective care and identifying the resource implications.

The bulletin and systematic review clearly covers significant clinical issues for practitioners. Evidence-Based Dentistry will be looking at some of the key areas in detail in future issues.

Enquiries concerning the bulletin should be addressed to NHS Centre for Reviews and Dissemination, University of York, York YO10 5DD; Telephone (+44) 1904 433634; Fax (+44) 1904) 433661: email revdis@york.ac.uk

\title{
Citation of articles in Evidence-based Dentistry
}

\section{Material from a structured abstract}

Raising lingual flaps increases the risk of nerve damage [Abstract]. Evidence-Based Dentistry 1998; 1: 14. Abstract of: Robinson P P, Smith K G. Lingual nerve damage during third molar removal: comparison of two surgical methods. Br Dent J 1996; 180: 456-461.

\section{Material from a commentary}

Creugars N. Commentary on: 'Two-thirds of fixed partial prostheses (bridges) last 15 years'. Evidence-Based Dentistry 1998; 1: 19. Comment on: Scurria M S, Bader J D, Shugars D A. Meta-analysis of fixed partial denture survival: prosthesis and abutments. J Prosthet Dent 1998; 79: 459-464.

Material published in the 'Editorial' and 'Toolbox' sections Richards D. Which journals should you read to keep up to date? Evidence-Based Dentistry 1998 November; 1: 22-25. 